\title{
Further Refinements of Jensen's Type Inequalities for the Function Defined on the Rectangle
}

\author{
M. Adil Khan, ${ }^{1}$ G. Ali Khan, ${ }^{1}$ T. Ali, ${ }^{1}$ T. Batbold, ${ }^{2}$ and A. Kiliçman ${ }^{3}$ \\ ${ }^{1}$ Department of Mathematics, University of Peshawar, Peshawar 25000, Pakistan \\ ${ }^{2}$ Institute of Mathematics, National University of Mongolia, P.O. Box 46A/104, 14201 Ulaanbaatar, Mongolia \\ ${ }^{3}$ Department of Mathematics and Institute of Mathematical Research, Universiti Putra Malaysia (UPM), \\ 43400 Serdang, Selangor, Malaysia
}

Correspondence should be addressed to A. Kiliçman; kilicman@yahoo.com

Received 26 September 2013; Accepted 10 November 2013

Academic Editor: Abdullah Alotaibi

Copyright (C) 2013 M. Adil Khan et al. This is an open access article distributed under the Creative Commons Attribution License, which permits unrestricted use, distribution, and reproduction in any medium, provided the original work is properly cited.

We give refinement of Jensen's type inequalities given by Bakula and Pečarić (2006) for the co-ordinate convex function. Also we establish improvement of Jensen's inequality for the convex function of two variables.

\section{Introduction}

Jensen's inequality for convex functions plays a crucial role in the theory of inequalities due to the fact that other inequalities such as the arithmetic mean-geometric mean inequality, the Hölder and Minkowski inequalities, and the Ky Fan inequality, can be obtained as particular cases of it. Therefore, it is worth studying it thoroughly and refining it from different point of view. There are many refinements of Jensen's inequality; see, for example, [1-14] and the references in them.

A function $f:[a, b] \times[c, d] \rightarrow \mathbb{R},[a, b] \times[c, d] \subset \mathbb{R}^{2}$ with $a<b$ and $c<d$ is called convex on the co-ordinates if the partial mappings $f_{y}:[a, b] \rightarrow \mathbb{R}$ defined as $f_{y}(t)=$ $f(t, y)$ and $f_{x}:[c, d] \rightarrow \mathbb{R}$ defined as $f_{x}(s)=f(x, s)$ are convex for all $x \in[a, b], y \in[c, d]$. Note that every convex function $f:[a, b] \times[c, d] \rightarrow \mathbb{R}$ is co-ordinate convex, but the converse is not generally true [8].

The following theorem has been given in [4].

Theorem 1. Let $f:[a, b] \times[c, d] \rightarrow \mathbb{R}$ be a convex function on the co-ordinates on $[a, b] \times[c, d]$. If $\mathbf{x}$ is an $n$-tuple in $[a, b]$, $\mathbf{y}$ is $m$-tuple in $[c, d], \mathbf{p}$ is a nonnegative $n$-tuple, and $\mathbf{w}$ is a nonnegative $m$-tuple such that $P_{n}=\sum_{i=1}^{n} p_{i}>0$ and $W_{m}=$ $\sum_{i=1}^{m} w_{j}>0$, then

$$
\begin{aligned}
f( & \left.\frac{1}{P_{n}} \sum_{i=1}^{n} p_{i} x_{i}, \frac{1}{W_{m}} \sum_{j=1}^{m} w_{j} y_{j}\right) \\
& \leq \frac{1}{2}\left\{\frac{1}{P_{n}} \sum_{i=1}^{n} p_{i} f\left(x_{i}, \bar{y}\right)+\frac{1}{W_{m}} \sum_{j=1}^{m} w_{j} f\left(\bar{x}, y_{j}\right)\right\} \\
& \leq \frac{1}{P_{n} W_{m}} \sum_{i=1}^{n} \sum_{j=1}^{m} p_{i} w_{j} f\left(x_{i}, y_{j}\right),
\end{aligned}
$$

where $\bar{x}=\left(1 / P_{n}\right) \sum_{i=1}^{n} p_{i} x_{i}$, and $\bar{y}=\left(1 / W_{m}\right) \sum_{j=1}^{m} w_{j} y_{j}$.

Recently Dragomir has given new refinement for Jensen inequality in [9]. The purpose of this paper is to give related refinements of Jensen's type inequalities (1) for the co-ordinate convex function. We will also discuss some particular interesting cases. We establish improvement of Jensen's inequality for the convex function defined on the rectangles. For related improvements of Jensen's inequality, 
see, for example, $[1,2,9,13,14]$. For further several related integral inequalities, see [15].

\section{Main Results}

Let $f:[a, b] \times[c, d] \rightarrow \mathbb{R}$ be convex on the co-ordinate on $[a, b] \times[c, d]$. If $x_{i} \in[a, b], y_{j} \in[c, d], p_{i}, w_{j}>0, i \in$ $\{1,2, \ldots, n\}, j \in\{1,2, \ldots, m\}$ with $P_{n}=\sum_{i=1}^{n} p_{i}$, and $W_{m}=$ $\sum_{j=1}^{m} w_{j}$, then for any subsets $I \subset\{1,2, \ldots, n\}$ and $J \subset$ $\{1,2, \ldots, m\}$, we assume that $\bar{I}:=\{1,2, \ldots, n\} \backslash I$ and $\bar{J}:=$ $\{1,2, \ldots, m\} \backslash J$. Define $P_{I}=\sum_{i \in I} p_{i}, P_{\bar{I}}=\sum_{i \in \bar{I}} p_{i}, W_{J}=$ $\sum_{j \in J} w_{j}$, and $W_{\bar{J}}=\sum_{j \in \bar{J}} w_{j}$. For the function $f$ and the $n$-, $m$-tuples, $\mathbf{x}=\left(x_{1}, x_{2}, \ldots, x_{n}\right), \mathbf{y}=\left(y_{1}, y_{2}, \ldots, y_{m}\right), \mathbf{p}=\left(p_{1}\right.$, $\left.p_{2}, \ldots, p_{n}\right)$, and $\mathbf{w}=\left(w_{1}, w_{2}, \ldots, w_{m}\right)$, we define the following functionals:

$D\left(f, \mathbf{p}, \mathbf{x}, I, y_{j}\right)$

$$
=\frac{P_{I}}{P_{n}} f\left(\frac{1}{P_{I}} \sum_{i \in I} p_{i} x_{i}, y_{j}\right)+\frac{P_{\bar{I}}}{P_{n}} f\left(\frac{1}{P_{\bar{I}}} \sum_{i \in \bar{I}} p_{i} x_{i}, y_{j}\right),
$$

$D\left(f, \mathbf{w}, \mathbf{y}, J, x_{i}\right)$

$$
=\frac{W_{J}}{W_{m}} f\left(x_{i}, \frac{1}{W_{J}} \sum_{j \in J} w_{j} y_{j}\right)+\frac{W_{\bar{J}}}{W_{m}} f\left(x_{i}, \frac{1}{W_{\bar{J}}} \sum_{j \in \bar{J}} w_{j} y_{j}\right),
$$

$D(f, \mathbf{p}, \mathbf{x}, I)$

$$
=\frac{P_{I}}{P_{n}} f\left(\frac{1}{P_{I}} \sum_{i \in I} p_{i} x_{i}, \bar{y}\right)+\frac{P_{\bar{I}}}{P_{n}} f\left(\frac{1}{P_{\bar{I}}} \sum_{i \in \bar{I}} p_{i} x_{i}, \bar{y}\right),
$$

$D(f, \mathbf{w}, \mathbf{y}, J)$

$$
=\frac{W_{J}}{W_{m}} f\left(\bar{x}, \frac{1}{W_{J}} \sum_{j \in J} w_{j} y_{j}\right)+\frac{W_{\bar{J}}}{W_{m}} f\left(\bar{x}, \frac{1}{W_{\bar{J}}} \sum_{j \in \bar{J}} w_{j} y_{j}\right),
$$

where $\bar{x}=\left(1 / P_{n}\right) \sum_{i=1}^{n} p_{i} x_{i}$, and $\bar{y}=\left(1 / W_{m}\right) \sum_{j=1}^{m} w_{j} y_{j}$.

It is worth to observe that for $I=\{k\}, k \in\{1, \ldots, n\}$, and $J=\{l\}, l \in\{1, \ldots, m\}$, we have the functionals

$$
\begin{aligned}
& D_{k}\left(f, \mathbf{p}, \mathbf{x}, y_{j}\right) \\
& \quad:=D\left(f, \mathbf{p}, \mathbf{x},\{k\}, y_{j}\right) \\
& \quad=\frac{p_{k}}{P_{n}} f\left(x_{k}, y_{j}\right)+\frac{P_{n}-p_{k}}{P_{n}} f\left(\frac{\sum_{i=1}^{n} p_{i} x_{i}-p_{k} x_{k}}{P_{n}-p_{k}}, y_{j}\right), \\
& D_{l}\left(f, \mathbf{w}, \mathbf{y}, x_{i}\right) \\
& \quad:=D\left(f, \mathbf{w}, \mathbf{y},\{l\}, x_{i}\right) \\
&=\frac{w_{l}}{W_{m}} f\left(x_{i}, y_{l}\right)+\frac{W_{m}-w_{l}}{W_{m}} f\left(x_{i}, \frac{\sum_{j=1}^{m} w_{j} y_{j}-w_{l} y_{l}}{W_{m}-w_{l}}\right),
\end{aligned}
$$

$$
\begin{aligned}
D_{k} & (f, \mathbf{p}, \mathbf{x}) \\
& :=D(f, \mathbf{p}, \mathbf{x},\{k\}) \\
& =\frac{p_{k}}{P_{n}} f\left(x_{k}, \bar{y}\right)+\frac{P_{n}-p_{k}}{P_{n}} f\left(\frac{\sum_{i=1}^{n} p_{i} x_{i}-p_{k} x_{k}}{P_{n}-p_{k}}, \bar{y}\right), \\
D_{l} & (f, \mathbf{w}, \mathbf{y}) \\
& :=D(f, \mathbf{w}, \mathbf{y},\{l\}) \\
& =\frac{w_{l}}{W_{m}} f\left(\bar{x}, y_{l}\right)+\frac{W_{m}-w_{l}}{W_{m}} f\left(\bar{x}, \frac{\sum_{j=1}^{m} w_{j} y_{j}-w_{l} y_{l}}{W_{m}-w_{l}}\right) .
\end{aligned}
$$

The following refinement of (1) holds.

Theorem 2. Let $f:[a, b] \times[c, d] \rightarrow \mathbb{R}$ be a co-ordinate convex function on $[a, b] \times[c, d]$. If $x_{i} \in[a, b], y_{j} \in[c, d], p_{i}$, $w_{j}>0, i \in\{1,2, \ldots, n\}, j \in\{1,2, \ldots, m\}$ with $P_{n}=\sum_{i=1}^{n} p_{i}$, and $W_{m}=\sum_{j=1}^{m} w_{j}$, then for any subsets $I \subset\{1,2, \ldots, n\}$ and $J \subset\{1,2, \ldots, m\}$, one has

$$
\begin{aligned}
& f(\bar{x}, \bar{y}) \\
& \leq \frac{1}{2}[D(f, \mathbf{w}, \mathbf{y}, J)+D(f, \mathbf{p}, \mathbf{x}, I)] \\
& \leq \frac{1}{2}\left[\frac{1}{P_{n}} \sum_{i=1}^{n} p_{i} f\left(x_{i}, \bar{y}\right)+\frac{1}{W_{m}} \sum_{j=1}^{m} w_{j} f\left(\bar{x}, y_{j}\right)\right] \\
& \leq \frac{1}{2}\left[\frac{1}{P_{n}} \sum_{i=1}^{n} p_{i} D\left(f, \mathbf{w}, \mathbf{y}, J, x_{i}\right)+\frac{1}{W_{m}} \sum_{j=1}^{m} w_{j} D\left(f, \mathbf{p}, \mathbf{x}, I, y_{j}\right)\right] \\
& \leq \frac{1}{P_{n} W_{m}} \sum_{i=1}^{n} \sum_{j=1}^{m} p_{i} w_{j} f\left(x_{i}, y_{j}\right),
\end{aligned}
$$

where $\bar{x}=\left(1 / P_{n}\right) \sum_{i=1}^{n} p_{i} x_{i}$, and $\bar{y}=\left(1 / W_{m}\right) \sum_{j=1}^{m} w_{j} y_{j}$.

Proof. One-dimensional Jensen's inequality gives us

$$
\begin{gathered}
f\left(x_{i}, \bar{y}\right) \leq \frac{1}{W_{m}} \sum_{j=1}^{m} w_{j} f\left(x_{i}, y_{j}\right), \\
f\left(\bar{x}, y_{j}\right) \leq \frac{1}{P_{n}} \sum_{i=1}^{n} p_{i} f\left(x_{i}, y_{j}\right) .
\end{gathered}
$$

As we have

$$
\begin{aligned}
& D\left(f, \mathbf{p}, \mathbf{x}, I, y_{j}\right) \\
& \quad=\frac{P_{I}}{P_{n}} f\left(\frac{1}{P_{I}} \sum_{i \in I} p_{i} x_{i}, y_{j}\right)+\frac{P_{\bar{I}}}{P_{n}} f\left(\frac{1}{P_{\bar{I}}} \sum_{i \in \bar{I}} p_{i} x_{i}, y_{j}\right),
\end{aligned}
$$


so by Jensen's inequality, we have

$$
\begin{aligned}
D\left(f, \mathbf{p}, \mathbf{x}, I, y_{j}\right) \\
=\frac{P_{I}}{P_{n}} f\left(\frac{1}{P_{I}} \sum_{i \in I} p_{i} x_{i}, y_{j}\right)+\frac{P_{\bar{I}}}{P_{n}} f\left(\frac{1}{P_{\bar{I}}} \sum_{i \in \bar{I}} p_{i} x_{i}, y_{j}\right) \\
\quad \leq \frac{P_{I}}{P_{n}} \frac{1}{P_{I}} \sum_{i \in I} p_{i} f\left(x_{i}, y_{j}\right)+\frac{P_{\bar{I}}}{P_{n}} \frac{1}{P_{\bar{I}}} \sum_{i \in \bar{I}} p_{i} f\left(x_{i}, y_{j}\right) \\
=\frac{1}{P_{n}} \sum_{i \in I} p_{i} f\left(x_{i}, y_{j}\right)+\frac{1}{P_{n}} \sum_{i \in \bar{I}} p_{i} f\left(x_{i}, y_{j}\right) \\
=\frac{1}{P_{n}} \sum_{i \in I \cup \bar{I}} p_{i} f\left(x_{i}, y_{j}\right) \\
\Longrightarrow D\left(f, \mathbf{p}, \mathbf{x}, I, y_{j}\right) \leq \frac{1}{P_{n}} \sum_{i=1}^{n} p_{i} f\left(x_{i}, y_{j}\right) .
\end{aligned}
$$

As the function $f$ is convex on the first co-ordinate, so we have

$$
\begin{aligned}
D & \left(f, \mathbf{p}, \mathbf{x}, I, y_{j}\right) \\
& =\frac{P_{I}}{P_{n}} f\left(\frac{1}{P_{I}} \sum_{i \in I} p_{i} x_{i}, y_{j}\right)+\frac{P_{\bar{I}}}{P_{n}} f\left(\frac{1}{P_{\bar{I}}} \sum_{i \in \bar{I}} p_{i} x_{i}, y_{j}\right) \\
& \geq f\left(\frac{P_{I}}{P_{n}} \frac{1}{P_{I}} \sum_{i \in I} p_{i} x_{i}+\frac{P_{\bar{I}}}{P_{n}} \frac{1}{P_{\bar{I}}} \sum_{i \in \bar{I}} p_{i} x_{i}, y_{j}\right) \\
& =f\left(\frac{1}{P_{n}} \sum_{i \in I} p_{i} x_{i}+\frac{1}{P_{n}} \sum_{i \in \bar{I}} p_{i} x_{i}, y_{j}\right) \\
& =f\left(\frac{1}{P_{n}} \sum_{i \in I \cup \bar{I}} p_{i} x_{i}, y_{j}\right) \\
& =f\left(\frac{1}{P_{n}} \sum_{i=1}^{n} p_{i} x_{i}, y_{j}\right) \\
& \Longrightarrow D\left(f, \mathbf{p}, \mathbf{x}, I, y_{j}\right) \geq f\left(\frac{1}{P_{n}} \sum_{i=1}^{n} p_{i} x_{i}, y_{j}\right) .
\end{aligned}
$$

Now, from (7) and (8), we have

$$
f\left(\bar{x}, y_{j}\right) \leq D\left(f, \mathbf{p}, \mathbf{x}, I, y_{j}\right) \leq \frac{1}{P_{n}} \sum_{i=1}^{n} p_{i} f\left(x_{i}, y_{j}\right) .
$$

Similarly, we can write

$$
f\left(x_{i}, \bar{y}\right) \leq D\left(f, \mathbf{w}, \mathbf{y}, J, x_{i}\right) \leq \frac{1}{W_{m}} \sum_{j=1}^{m} w_{j} f\left(x_{i}, y_{j}\right)
$$

Multiplying (9) and (10), respectively, by $w_{j}$ and $p_{i}$ and summing over $i$ and $j$, we obtain

$$
\begin{aligned}
\frac{1}{W_{m}} \sum_{j=1}^{m} w_{j} f\left(\bar{x}, y_{j}\right) & \leq \frac{1}{W_{m}} \sum_{j=1}^{m} w_{j} D\left(f, \mathbf{p}, \mathbf{x}, I, y_{j}\right) \\
& \leq \frac{1}{P_{n} W_{m}} \sum_{i=1}^{n} \sum_{j=1}^{m} p_{i} w_{j} f\left(x_{i}, y_{j}\right), \\
\frac{1}{P_{n}} \sum_{i=1}^{n} p_{i} f\left(x_{i}, \bar{y}\right) \leq & \frac{1}{P_{n}} \sum_{i=1}^{n} p_{i} D\left(f, \mathbf{w}, \mathbf{y}, J, x_{i}\right) \\
& \leq \frac{1}{P_{n} W_{m}} \sum_{i=1}^{n} \sum_{j=1}^{m} p_{i} w_{j} f\left(x_{i}, y_{j}\right) .
\end{aligned}
$$

Adding (11) and (12), we have

$$
\begin{gathered}
\frac{1}{2}\left[\frac{1}{P_{n}} \sum_{i=1}^{n} p_{i} f\left(x_{i}, \bar{y}\right)+\frac{1}{W_{m}} \sum_{j=1}^{m} w_{j} f\left(\bar{x}, y_{j}\right)\right] \\
\leq \frac{1}{2}\left[\frac{1}{P_{n}} \sum_{i=1}^{n} p_{i} D\left(f, \mathbf{w}, \mathbf{y}, J, x_{i}\right)\right. \\
\left.+\frac{1}{W_{m}} \sum_{j=1}^{m} w_{j} D\left(f, \mathbf{p}, \mathbf{x}, I, y_{j}\right)\right] \\
\leq \frac{1}{P_{n} W_{m}} \sum_{i=1}^{n} \sum_{j=1}^{m} p_{i} w_{j} f\left(x_{i}, y_{j}\right) .
\end{gathered}
$$

Again by one-dimensional Jensen's inequality, we have

$$
\begin{gathered}
f(\bar{x}, \bar{y}) \leq \frac{1}{P_{n}} \sum_{i=1}^{n} p_{i} f\left(x_{i}, \bar{y}\right), \\
f(\bar{x}, \bar{y}) \leq \frac{1}{W_{m}} \sum_{j=1}^{m} w_{j} f\left(\bar{x}, y_{j}\right) .
\end{gathered}
$$

As we have the functional

$$
D(f, \mathbf{p}, \mathbf{x}, I)=\frac{P_{I}}{P_{n}} f\left(\frac{1}{P_{I}} \sum_{i \in I} p_{i} x_{i}, \bar{y}\right)+\frac{P_{\bar{I}}}{P_{n}} f\left(\frac{1}{P_{\bar{I}}} \sum_{i \in \bar{I}} p_{i} x_{i}, \bar{y}\right),
$$

so by Jensen's inequality, we get

$$
\begin{aligned}
D(f, \mathbf{p}, \mathbf{x}, I)= & \frac{P_{I}}{P_{n}} f\left(\frac{1}{P_{I}} \sum_{i \in I} p_{i} x_{i}, \bar{y}\right) \\
& +\frac{P_{\bar{I}}}{P_{n}} f\left(\frac{1}{P_{\bar{I}}} \sum_{i \in \bar{I}} p_{i} x_{i}, \bar{y}\right) \\
\leq & \frac{P_{I}}{P_{n}} \frac{1}{P_{I}} \sum_{i \in I} p_{i} f\left(x_{i}, \bar{y}\right)+\frac{P_{\bar{I}}}{P_{n}} \frac{1}{P_{\bar{I}}} \sum_{i \in \bar{I}} p_{i} f\left(x_{i}, \bar{y}\right)
\end{aligned}
$$




$$
\begin{aligned}
& =\frac{1}{P_{n}} \sum_{i \in I} p_{i} f\left(x_{i}, \bar{y}\right)+\frac{1}{P_{n}} \sum_{i \in \bar{I}} p_{i} f\left(x_{i}, \bar{y}\right) \\
& =\frac{1}{P_{n_{i \in I}}} \sum_{\bar{I}} p_{i} f\left(x_{i}, \bar{y}\right) \\
& \Longrightarrow D(f, \mathbf{p}, \mathbf{x}, I) \leq \frac{1}{P_{n}} \sum_{i=1}^{n} p_{i} f\left(x_{i}, \bar{y}\right),
\end{aligned}
$$

and as the function $f$ is convex on the first co-ordinate, so we have

$$
\begin{aligned}
D(f, \mathbf{p}, \mathbf{x}, I)= & \frac{P_{I}}{P_{n}} f\left(\frac{1}{P_{I}} \sum_{i \in I} p_{i} x_{i}, \bar{y}\right) \\
& +\frac{P_{\bar{I}}}{P_{n}} f\left(\frac{1}{P_{\bar{I}}} \sum_{i \in \bar{I}} p_{i} x_{i}, \bar{y}\right) \\
\geq & f\left(\frac{P_{I}}{P_{n}} \frac{1}{P_{I}} \sum_{i \in I} p_{i} x_{i}+\frac{P_{\bar{I}}}{P_{n}} \frac{1}{P_{\bar{I}}} \sum_{i \in \bar{I}} p_{i} x_{i}, \bar{y}\right) \\
= & f\left(\frac{1}{P_{n}} \sum_{i \in I} p_{i} x_{i}+\frac{1}{P_{n}} \sum_{i \in \bar{I}} p_{i} x_{i}, \bar{y}\right) \\
= & f\left(\frac{1}{P_{n}} \sum_{i \in I \cup \bar{I}} p_{i} x_{i}, \bar{y}\right)=f\left(\frac{1}{P_{n}} \sum_{i=1}^{n} p_{i} x_{i}, \bar{y}\right) \\
& \Longrightarrow D(f, \mathbf{p}, \mathbf{x}, I) \geq f(\bar{x}, \bar{y}) .
\end{aligned}
$$

Now from (16) and (17), we have

$$
f(\bar{x}, \bar{y}) \leq D(f, \mathbf{p}, \mathbf{x}, I) \leq \frac{1}{P_{n}} \sum_{i=1}^{n} p_{i} f\left(x_{i}, \bar{y}\right) .
$$

Similarly, we can prove that

$$
f(\bar{x}, \bar{y}) \leq D(f, \mathbf{w}, \mathbf{y}, J) \leq \frac{1}{W_{m}} \sum_{j=1}^{m} w_{j} f\left(\bar{x}, y_{j}\right)
$$

Adding (18) and (19), we get

$$
\begin{aligned}
f(\bar{x}, \bar{y}) & \leq \frac{1}{2}[D(f, \mathbf{w}, \mathbf{y}, J)+D(f, \mathbf{p}, \mathbf{x}, I)] \\
& \leq \frac{1}{2}\left[\frac{1}{P_{n}} \sum_{i=1}^{n} p_{i} f\left(x_{i}, \bar{y}\right)+\frac{1}{W_{m}} \sum_{j=1}^{m} w_{j} f\left(\bar{x}, y_{j}\right)\right] .
\end{aligned}
$$

Combining (13) and (20), we have

$$
\begin{aligned}
& f(\bar{x}, \bar{y}) \\
& \leq \frac{1}{2}[D(f, \mathbf{w}, \mathbf{y}, J)+D(f, \mathbf{p}, \mathbf{x}, I)] \\
& \leq \frac{1}{2}\left[\frac{1}{P_{n}} \sum_{i=1}^{n} p_{i} f\left(x_{i}, \bar{y}\right)+\frac{1}{W_{m}} \sum_{j=1}^{m} w_{j} f\left(\bar{x}, y_{j}\right)\right] \\
& \leq \frac{1}{2}\left[\frac{1}{P_{n}} \sum_{i=1}^{n} p_{i} D\left(f, \mathbf{w}, \mathbf{y}, J, x_{i}\right)\right. \\
& \left.\quad+\frac{1}{W_{m}} \sum_{j=1}^{m} w_{j} D\left(f, \mathbf{p}, \mathbf{x}, I, y_{j}\right)\right] \\
& \leq \frac{1}{P_{n} W_{m}} \sum_{i=1}^{n} \sum_{j=1}^{m} p_{i} w_{j} f\left(x_{i}, y_{j}\right) .
\end{aligned}
$$

The following cases from the above inequalities are of interest $[6,7]$.

Remark 3. We observe that the inequalities in (4) can be written equivalently as

$$
\frac{1}{P_{n} W_{m}} \sum_{i=1}^{n} \sum_{j=1}^{m} p_{i} w_{j} f\left(x_{i}, y_{j}\right)
$$

$$
\begin{aligned}
\geq \max _{\substack{I \subset\{11, \ldots, n\} \\
J \subset\{1, \ldots, m\}}} \frac{1}{2}\left[\frac{1}{P_{n}} \sum_{i=1}^{n} p_{i} D\left(f, \mathbf{w}, \mathbf{y}, J, x_{i}\right)\right. \\
\left.+\frac{1}{W_{m}} \sum_{j=1}^{m} w_{j} D\left(f, \mathbf{p}, \mathbf{x}, I, y_{j}\right)\right],
\end{aligned}
$$

$$
\begin{array}{r}
\frac{1}{P_{n}} \sum_{i=1}^{n} p_{i} f\left(x_{i}, \bar{y}\right)+\frac{1}{W_{m}} \sum_{j=1}^{m} w_{j} f\left(\bar{x}, y_{j}\right) \\
\leq \min _{\substack{I \subset\{1, \ldots, n\} \\
J \subset\{1, \ldots, m\}}}\left[\frac{1}{P_{n}} \sum_{i=1}^{n} p_{i} D\left(f, \mathbf{w}, \mathbf{y}, J, x_{i}\right)\right.
\end{array}
$$

$$
\begin{array}{r}
\left.+\frac{1}{W_{m}} \sum_{j=1}^{m} w_{j} D\left(f, \mathbf{p}, \mathbf{x}, I, y_{j}\right)\right], \\
\frac{1}{P_{n}} \sum_{i=1}^{n} p_{i} f\left(x_{i}, \bar{y}\right)+\frac{1}{W_{m}} \sum_{j=1}^{m} w_{j} f\left(\bar{x}, y_{j}\right) \\
\geq \max _{\substack{I \subset\{11, \ldots, n\} \\
J \subset\{1, \ldots, m\}}}[D(f, \mathbf{w}, \mathbf{y}, J)+D(f, \mathbf{p}, \mathbf{x}, I)],
\end{array}
$$




$$
\begin{aligned}
& f(\bar{x}, \bar{y}) \\
& \quad \leq \min _{\substack{I \subset\{1, \ldots, n\} \\
J \subset\{1, \ldots, m\}}} \frac{1}{2}[D(f, \mathbf{w}, \mathbf{y}, J)+D(f, \mathbf{p}, \mathbf{x}, I)] .
\end{aligned}
$$

These inequalities imply the following results:

$$
\begin{aligned}
& \frac{1}{P_{n} W_{m}} \sum_{i=1}^{n} \sum_{j=1}^{m} p_{i} w_{j} f\left(x_{i}, y_{j}\right) \\
& \geq \max _{\substack{k \in\{1, \ldots, n\} \\
l \in\{1, \ldots, m\}}} \frac{1}{2}\left[\frac{1}{P_{n}} \sum_{i=1}^{n} p_{i} D_{l}\left(f, \mathbf{w}, \mathbf{y}, x_{i}\right)\right. \\
& \left.+\frac{1}{W_{m}} \sum_{j=1}^{m} w_{j} D_{k}\left(f, \mathbf{p}, \mathbf{x}, y_{j}\right)\right], \\
& \frac{1}{P_{n}} \sum_{i=1}^{n} p_{i} f\left(x_{i}, \bar{y}\right)+\frac{1}{W_{m}} \sum_{j=1}^{m} w_{j} f\left(\bar{x}, y_{j}\right) \\
& \leq \min _{\substack{k \in\{1, \ldots, n\} \\
l \in\{1, \ldots, m\}}}\left[\frac{1}{P_{n}} \sum_{i=1}^{n} p_{i} D_{l}\left(f, \mathbf{w}, \mathbf{y}, x_{i}\right)\right. \\
& \left.+\frac{1}{W_{m}} \sum_{j=1}^{m} w_{j} D_{k}\left(f, \mathbf{p}, \mathbf{x}, y_{j}\right)\right], \\
& \frac{1}{P_{n}} \sum_{i=1}^{n} p_{i} f\left(x_{i}, \bar{y}\right)+\frac{1}{W_{m}} \sum_{j=1}^{m} w_{j} f\left(\bar{x}, y_{j}\right) \\
& \geq \max _{\substack{k \in\{1, \ldots, n\} \\
l \in\{1, \ldots, m\}}}\left[D_{l}(f, \mathbf{w}, \mathbf{y})+D_{k}(f, \mathbf{p}, \mathbf{x})\right], \\
& f(\bar{x}, \bar{y}) \leq \min _{\substack{k \in\{1, \ldots, n\} \\
l \in\{1, \ldots, m\}}} \frac{1}{2}\left[D_{l}(f, \mathbf{w}, \mathbf{y})+D_{k}(f, \mathbf{p}, \mathbf{x})\right] .
\end{aligned}
$$

Moreover, from the above, we also have

$$
\begin{aligned}
& \max _{I \subset\{1, \ldots, n\}} D(f, \mathbf{p}, \mathbf{x} ; I) \geq \max _{k \in\{1, \ldots, n\}} D_{k}(f, \mathbf{p}, \mathbf{x}), \\
& \max _{J \subset\{1, \ldots, m\}} D(f, \mathbf{w}, \mathbf{y} ; J) \geq \max _{l \in\{1, \ldots, m\}} D_{l}(f, \mathbf{w}, \mathbf{y}), \\
& \max _{I \subset\{1, \ldots, n\}} D\left(f, \mathbf{p}, \mathbf{x} ; I, y_{j}\right) \geq \max _{k \in\{1, \ldots, n\}} D_{k}(f, \mathbf{p}, \mathbf{x}, \mathbf{y} j), \\
& \max _{J \subset\{1, \ldots, m\}} D\left(f, \mathbf{w}, \mathbf{y} ; J, x_{i}\right) \geq \max _{l \in\{1, \ldots, m\}} D_{l}\left(f, \mathbf{w}, \mathbf{y}, x_{i}\right), \\
& \min _{k \in\{1, \ldots, n\}} D_{k}(f, \mathbf{p}, \mathbf{x}) \geq \min _{I \subset\{1, \ldots, n\}} D(f, \mathbf{p}, \mathbf{x} ; I), \\
& \min _{l \in\{1, \ldots, m\}} D_{l}(f, \mathbf{w}, \mathbf{y}) \geq \min _{J \subset\{1, \ldots, m\}} D(f, \mathbf{w}, \mathbf{y} ; J),
\end{aligned}
$$

$$
\begin{aligned}
& \min _{k \in\{1, \ldots, n\}} D_{k}\left(f, \mathbf{p}, \mathbf{x}, y_{j}\right) \geq \min _{I \subset\{1, \ldots, n\}} D\left(f, \mathbf{p}, \mathbf{x} ; I, y_{j}\right), \\
& \min _{l \in\{1, \ldots, m\}} D_{l}\left(f, \mathbf{w}, \mathbf{y}, x_{i}\right) \geq \min _{J \subset\{1, \ldots, m\}} D\left(f, \mathbf{w}, \mathbf{y} ; J, x_{i}\right) .
\end{aligned}
$$

We discuss the following particular cases of the above inequalities which is of interest [6].

In the case when $p_{i}=1$ and $w_{j}=1$ for $i \in\{1, \ldots, n\}$ and $j \in\{1, \ldots, m\}$, consider the natural numbers $k, l$ with $1 \leq k \leq n-1$ and $1 \leq l \leq m-1$ and define

$$
\begin{array}{r}
D_{k}\left(f, \mathbf{x}, y_{j}\right) \\
\quad=\frac{k}{n} f\left(\frac{1}{k} \sum_{i=1}^{k} x_{i}, y_{j}\right)+\frac{n-k}{n} f\left(\frac{1}{n-k} \sum_{i=k+1}^{n} x_{i}, y_{j}\right), \\
D_{l}\left(f, \mathbf{y}, x_{i}\right) \\
=\frac{l}{m} f\left(x_{i}, \frac{1}{l} \sum_{j=1}^{l} y_{j}\right)+\frac{m-l}{m} f\left(x_{i}, \frac{1}{m-l} \sum_{j=l+1}^{m} y_{j}\right), \\
D_{k}(f, \mathbf{x})=\frac{k}{n} f\left(\frac{1}{k} \sum_{i=1}^{k} x_{i}, \bar{y}\right)+\frac{n-k}{n} f\left(\frac{1}{n-k} \sum_{i=k+1}^{n} x_{i}, \bar{y}\right), \\
D_{l}(f, \mathbf{y})=\frac{l}{m} f\left(\bar{x}, \frac{1}{l} \sum_{j=1}^{l} y_{j}\right)+\frac{m-l}{m} f\left(\bar{x}, \frac{1}{m-l} \sum_{j=l+1}^{m} y_{j}\right) .
\end{array}
$$

We can give the following result.

Corollary 4. Let $f:[a, b] \times[c, d] \rightarrow \mathbb{R}$ be a co-ordinate convex function on $[a, b] \times[c, d]$. If $x_{i} \in[a, b]$ and $y_{j} \in[c, d]$, then for any $k \in\{1, \ldots, n-1\}$ and $l \in\{1, \ldots, m-1\}$, one has

$$
\begin{aligned}
f\left(\frac{1}{n} \sum_{i=1}^{n} x_{i}, \frac{1}{m} \sum_{j=1}^{m} y_{j}\right) \\
\quad \leq \frac{1}{2}\left[D_{l}(f, \mathbf{y})+D_{k}(f, \mathbf{x})\right] \\
\quad \leq \frac{1}{2}\left[\frac{1}{n} \sum_{i=1}^{n} f\left(x_{i}, \bar{y}\right)+\frac{1}{m} \sum_{j=1}^{m} f\left(\bar{x}, y_{j}\right)\right] \\
\quad \leq \frac{1}{2}\left[\frac{1}{n} \sum_{i=1}^{n} D_{l}\left(f, \mathbf{y}, x_{i}\right)+\frac{1}{m} \sum_{j=1}^{m} D_{k}\left(f, \mathbf{x}, y_{j}\right)\right] \\
\leq \frac{1}{m n} \sum_{i=1}^{n} \sum_{j=1}^{m} f\left(x_{i}, y_{j}\right) .
\end{aligned}
$$


In particular, we have the bounds

$$
\begin{gathered}
\frac{1}{m n} \sum_{i=1}^{n} \sum_{j=1}^{m} f\left(x_{i}, y_{j}\right) \\
\geq \max _{\substack{k \in\{1, \ldots, n\} \\
l \in\{1, \ldots, m\}}} \frac{1}{2}\left[\frac{1}{n} \sum_{i=1}^{n} D_{l}\left(f, \mathbf{y}, x_{i}\right)\right. \\
\left.+\frac{1}{m} \sum_{j=1}^{m} D_{k}\left(f, \mathbf{x}, y_{j}\right)\right], \\
\frac{1}{n} \sum_{i=1}^{n} f\left(x_{i}, \bar{y}\right)+\frac{1}{m} \sum_{j=1}^{m} f\left(\bar{x}, y_{j}\right) \\
\leq \min _{\substack{k \in\{1, \ldots, n\} \\
l \in\{1, \ldots, m\}}}\left[\frac{1}{n} \sum_{i=1}^{n} D_{l}\left(f, \mathbf{y}, x_{i}\right)\right. \\
\left.+\frac{1}{m} \sum_{j=1}^{m} D_{k}\left(f, \mathbf{x}, y_{j}\right)\right], \\
\frac{1}{n} \sum_{i=1}^{n} f\left(x_{i}, \bar{y}\right)+\frac{1}{m} \sum_{j=1}^{m} f\left(\bar{x}, y_{j}\right) \\
\geq \max _{\substack{k \in\{1, \ldots, n\} \\
l \in\{1, \ldots, m\}}}\left[D_{l}(f, \mathbf{y})+D_{k}(f, \mathbf{x})\right], \\
\min _{\substack{k \in\{1, \ldots, n\} \\
l \in\{1, \ldots, m\}}} \frac{1}{2}\left[D_{l}(f, \mathbf{y})+D_{k}(f, \mathbf{x})\right] .
\end{gathered}
$$

Remark 5. Note that if we substitute $m=1, f\left(x, y_{1}\right) \rightarrow$ $f(x), D\left(f, \mathbf{w}, \mathbf{y}, J, x_{i}\right)=f(\bar{x}), D\left(f, \mathbf{p}, \mathbf{x}, I, y_{j}\right)=D(f, \mathbf{p}, \mathbf{x}, I)$, and $D(f, \mathbf{w}, \mathbf{y}, J)=f(\bar{x})$ in Theorem 2, we get the following result of Dragomir [9] for convex function defined on the interval and $\sum_{i=1}^{n} p_{i}=P_{n}>0$,

$$
\begin{aligned}
f\left(\frac{1}{P_{n}} \sum_{i=1}^{n} p_{i} x_{i}\right) & \leq \frac{P_{I}}{P_{n}} f\left(\frac{1}{P_{I}} \sum_{i \in I} p_{i} x_{i}\right)+\frac{P_{\bar{I}}}{P_{n}} f\left(\frac{1}{P_{\bar{I}}} \sum_{i \in \bar{I}} p_{i} x_{i}\right) \\
& \leq \frac{1}{P_{n}} \sum_{i=1}^{n} p_{i} f\left(x_{i}\right) .
\end{aligned}
$$

The following refinement of Hölder inequality holds.

Corollary 6. Let $\mathbf{x}=\left(x_{1}, x_{2}, \ldots, x_{n}\right)$ and $\mathbf{y}=\left(y_{1}, y_{2}, \ldots, y_{n}\right)$ be two positive $n$-tuples. Then for $(1 / p)+(1 / q)=1, p, q>1$, one has

$$
\begin{aligned}
& \sum_{i=1}^{n} x_{i} y_{i} \\
& \leq\left[( \sum _ { i = 1 } ^ { n } y _ { i } ^ { q } ) ^ { p / q } \left\{\left(\sum_{i \in I} y_{i}^{q}\right)^{-p / q}\left(\sum_{i \in I} x_{i} y_{i}\right)^{p}\right.\right. \\
& \left.\left.+\left(\sum_{i \in \bar{I}} y_{i}^{q}\right)^{-p / q}\left(\sum_{i \in \bar{I}} x_{i} y_{i}\right)^{p}\right\}\right]^{1 / p} \\
& \leq\left(\sum_{i=1}^{n} x_{i}^{p}\right)^{1 / p}\left(\sum_{i=1}^{n} y_{i}^{q}\right)^{1 / q} .
\end{aligned}
$$

Proof. Using the functions $f(x)=x^{p}, p>1, x_{i} \rightarrow x_{i} y_{i}^{-q / p}$, and $p_{i} \rightarrow y_{i}^{q}$ in (28), we get (29).

Remark 7. As mentioned above from the inequalities in (29), we can write

$$
\begin{aligned}
& \left(\sum_{i=1}^{n} x_{i}^{p}\right)^{1 / p}\left(\sum_{i=1}^{n} y_{i}^{q}\right)^{1 / q} \\
& \geq \max _{I \subset\{1, \ldots, n\}}\left[( \sum _ { i = 1 } ^ { n } y _ { i } ^ { q } ) ^ { p / q } \left\{\left(\sum_{i \in I} y_{i}^{q}\right)^{-p / q}\left(\sum_{i \in I} x_{i} y_{i}\right)^{p}\right.\right. \\
& \left.\left.+\left(\sum_{i \in \bar{I}} y_{i}^{q}\right)^{-p / q}\left(\sum_{i \in \bar{I}} x_{i} y_{i}\right)^{p}\right\}\right]^{1 / p}, \\
& \sum_{i=1}^{n} x_{i} y_{i} \\
& \leq \min _{I \subset\{1, \ldots, n\}}\left[( \sum _ { i = 1 } ^ { n } y _ { i } ^ { q } ) ^ { p / q } \left\{\left(\sum_{i \in I} y_{i}^{q}\right)^{-p / q}\left(\sum_{i \in I} x_{i} y_{i}\right)^{p}\right.\right. \\
& \left.\left.+\left(\sum_{i \in \bar{I}} y_{i}^{q}\right)^{-p / q}\left(\sum_{i \in \bar{I}} x_{i} y_{i}\right)^{p}\right\}\right]^{1 / p} .
\end{aligned}
$$

The following improvement of Jensen's inequality is valid.

Theorem 8. Let $f:[a, b] \times[c, d] \rightarrow \mathbb{R}$ be convex on the coordinates of $[a, b] \times[c, d] \subseteq \mathbb{R}^{2}$. If $\mathbf{x}$ is an n-tuple in $[a, b], \mathbf{y}$ is an $m$-tuple in $[c, d], \mathbf{p}$ is a nonnegative $n$-tuple such that $P_{n}=$ $\sum_{i=1}^{n} p_{i}>0$, and $\mathbf{w}$ is a nonnegative $m$-tuple such that $W_{m}=$ $\sum_{j=1}^{m} w_{j}>0$, then

$$
\begin{aligned}
& \frac{1}{P_{n} W_{m}} \sum_{i=1}^{n} \sum_{j=1}^{m} p_{i} w_{j} f\left(x_{i}, y_{j}\right)-f(\bar{x}, \bar{y}) \\
& \geq\left|\frac{1}{P_{n} W_{m}} \sum_{i=1}^{n} \sum_{j=1}^{m} p_{i} w_{j}\right| f\left(x_{i}, y_{j}\right)-f(\bar{x}, \bar{y}) \mid \\
& \quad-\frac{1}{P_{n} W_{m}} \sum_{i=1}^{n} \sum_{j=1}^{m} p_{i} w_{j} \mid \frac{\partial f_{+}(\bar{x}, \bar{y})}{\partial w}\left(x_{i}-\bar{x}\right) \\
& +\frac{\partial f_{+}(\bar{x}, \bar{y})}{\partial z}\left(y_{j}-\bar{y}\right)||,
\end{aligned}
$$

where $\bar{x}=\left(1 / P_{n}\right) \sum_{i=1}^{n} p_{i} x_{i}$, and $\bar{y}=\left(1 / W_{m}\right) \sum_{j=1}^{m} w_{j} y_{j}$.

Proof. Since $f$ is convex on $[a, b] \times[c, d]$, therefore we have

$$
\begin{aligned}
& f(x, y)-f(w, z) \\
& \quad \geq \frac{\partial f_{+}(w, z)}{\partial w}(x-w)+\frac{\partial f_{+}(w, z)}{\partial z}(y-z) .
\end{aligned}
$$


From the above inequality, we have

$$
\begin{aligned}
f(x, y) & -f(w, z)-\frac{\partial f_{+}(w, z)}{\partial w}(x-w)-\frac{\partial f_{+}(w, z)}{\partial z}(y-z) \\
= & \mid f(x, y)-f(w, z)-\frac{\partial f_{+}(w, z)}{\partial w}(x-w) \\
& -\frac{\partial f_{+}(w, z)}{\partial z}(y-z) \mid \\
\geq & || f(x, y)-f(w, z) \mid \\
& -\left|\frac{\partial f_{+}(w, z)}{\partial w}(x-w)+\frac{\partial f_{+}(w, z)}{\partial z}(y-z)\right| \mid .
\end{aligned}
$$

Let $x \rightarrow x_{i}, y \rightarrow y_{j}, w \rightarrow \sum_{i=1}^{n} p_{i} x_{i} / P_{n}:=\bar{x}$, and $z \rightarrow$ $\sum_{j=1}^{n} w_{j} y_{j} / W_{m}:=\bar{y}$, then (33) becomes

$$
\begin{aligned}
& f\left(x_{i}, y_{j}\right)-f(\bar{x}, \bar{y}) \\
& \quad-\frac{\partial f_{+}(\bar{x}, \bar{y})}{\partial w}\left(x_{i}-\bar{x}\right)-\frac{\partial f_{+}(\bar{x}, \bar{y})}{\partial z}\left(y_{j}-\bar{y}\right) \\
& \geq|| f\left(x_{i}, y_{j}\right)-f(\bar{x}, \bar{y}) \mid \\
& \quad-\left|\frac{\partial f_{+}(\bar{x}, \bar{y})}{\partial w}\left(x_{i}-\bar{x}\right)+\frac{\partial f_{+}(\bar{x}, \bar{y})}{\partial z}\left(y_{j}-\bar{y}\right)\right| \mid
\end{aligned}
$$

Multiplying (34) by $p_{i}$ and $w_{j}$ and summing over $i$ and $j$, we have

$$
\begin{gathered}
\sum_{i=1}^{n} \sum_{j=1}^{m} p_{i} w_{j} f\left(x_{i}, y_{j}\right)-\sum_{i=1}^{n} \sum_{j=1}^{m} p_{i} w_{j} f(\bar{x}, \bar{y}) \\
\quad-\sum_{i=1}^{n} \sum_{j=1}^{m} p_{i} w_{j} \frac{\partial f_{+}(\bar{x}, \bar{y})}{\partial w}\left(x_{i}-\bar{x}\right) \\
\quad-\sum_{i=1}^{n} \sum_{j=1}^{m} p_{i} w_{j} \frac{\partial f_{+}(\bar{x}, \bar{y})}{\partial z}\left(y_{j}-\bar{y}\right) \\
\geq\left|\sum_{i=1}^{n} \sum_{j=1}^{m} p_{i} w_{j}\right| f\left(x_{i}, y_{j}\right)-f(\bar{x}, \bar{y}) \mid \\
\quad-\sum_{i=1}^{n} \sum_{j=1}^{m} p_{i} w_{j} \mid \frac{\partial f_{+}(\bar{x}, \bar{y})}{\partial w}\left(x_{i}-\bar{x}\right) \\
+\frac{\partial f_{+}(\bar{x}, \bar{y})}{\partial z}\left(y_{j}-\bar{y}\right)|| .
\end{gathered}
$$

One has

$$
\begin{gathered}
\sum_{i=1}^{n} \sum_{j=1}^{m} p_{i} w_{j} \frac{\partial f_{+}(\bar{x}, \bar{y})}{\partial w}\left(x_{i}-\bar{x}\right) \\
=\sum_{j=1}^{m} w_{j} \frac{\partial f_{+}(\bar{x}, \bar{y})}{\partial w}\left(\sum_{i=1}^{n} p_{i} x_{i}-P_{n} \cdot \frac{1}{P_{n}} \sum_{i=1}^{n} p_{i} x_{i}\right)=0, \\
\sum_{i=1}^{n} \sum_{j=1}^{m} p_{i} w_{j} \frac{\partial f_{+}(\bar{x}, \bar{y})}{\partial z}\left(y_{j}-\bar{y}\right)=0 .
\end{gathered}
$$

Therefore (35) becomes

$$
\begin{gathered}
\sum_{i=1}^{n} \sum_{j=1}^{m} p_{i} w_{j} f\left(x_{i}, y_{j}\right)-\sum_{i=1}^{n} \sum_{j=1}^{m} p_{i} w_{j} f(\bar{x}, \bar{y}) \\
\geq\left|\sum_{i=1}^{n} \sum_{j=1}^{m} p_{i} w_{j}\right| f\left(x_{i}, y_{j}\right)-f(\bar{x}, \bar{y}) \mid \\
-\sum_{i=1}^{n} \sum_{j=1}^{m} p_{i} w_{j} \mid \frac{\partial f_{+}(\bar{x}, \bar{y})}{\partial w}\left(x_{i}-\bar{x}\right) \\
+\frac{\partial f_{+}(\bar{x}, \bar{y})}{\partial z}\left(y_{j}-\bar{y}\right)|| .
\end{gathered}
$$

Multiplying both hand sides by $1 / P_{n} W_{m}$, we have

$$
\begin{aligned}
& \frac{1}{P_{n} W_{m}} \sum_{i=1}^{n} \sum_{j=1}^{m} p_{i} w_{j} f\left(x_{i}, y_{j}\right)-f(\bar{x}, \bar{y}) \\
& \geq\left|\frac{1}{P_{n} W_{m}} \sum_{i=1}^{n} \sum_{j=1}^{m} p_{i} w_{j}\right| f\left(x_{i}, y_{j}\right)-f(\bar{x}, \bar{y}) \mid \\
& \quad-\frac{1}{P_{n} W_{m}} \sum_{i=1}^{n} \sum_{j=1}^{m} p_{i} w_{j} \mid \frac{\partial f_{+}(\bar{x}, \bar{y})}{\partial w}\left(x_{i}-\bar{x}\right) \\
& +\frac{\partial f_{+}(\bar{x}, \bar{y})}{\partial z}\left(y_{j}-\bar{y}\right)|| .
\end{aligned}
$$

This completes the proof.

\section{Conflict of Interests}

The authors declare that they have no conflict of interests regarding publication of this paper.

\section{Acknowledgments}

The authors are grateful to the referees for the useful comments regarding presentation in the early version of the paper. The last author also acknowledges that the present work was partially supported by the University Putra Malaysia (UPM).

\section{References}

[1] M. Adil Khan, M. Anwar, J. Jakšetić, and J. Pečarić, "On some improvements of the Jensen inequality with some applications," 
Journal of Inequalities and Applications, vol. 2009, Article ID 323615, 15 pages, 2009.

[2] M. Adil Khan, S. Khalid, and J. Pečarić, "Improvement of Jensen's inequality in terms of Gâteaux derivatives for convex functions in linear spaces with applications," Kyungpook Mathematical Journal, vol. 52, no. 4, pp. 495-511, 2012.

[3] M. A. Khan, S. Khalid, and J. Pečarić, "Refinements of some majorization type inequalities," Journal of Mathematical Inequalities, vol. 7, no. 1, pp. 73-92, 2013.

[4] M. Klaričić Bakula and J. Pečarić, “On the Jensen's inequality for convex functions on the co-ordinates in a rectangle from the plane," Taiwanese Journal of Mathematics, vol. 10, no. 5, pp. 1271-1292, 2006.

[5] L. Horváth, "A parameter-dependent refinement of the discrete Jensen's inequality for convex and mid-convex functions," Journal of Inequalities and Applications, vol. 2011, article 26, 2011.

[6] S. S. Dragomir, "A refinement of Jensen's inequality with applications for f-divergence measures," Taiwanese Journal of Mathematics, vol. 14, no. 1, pp. 153-164, 2010.

[7] S. S. Dragomir, "Some refinements of Jensen's inequality," Journal of Mathematical Analysis and Applications, vol. 168, no. 2, pp. 518-522, 1992.

[8] S. S. Dragomir, "On Hadamard's inequality for the convex mappings defined on a ball in the space and applications," Mathematical Inequalities \& Applications, vol. 3, no. 2, pp. 177187,2000

[9] S. S. Dragomir, “A new refinement of Jensen's inequality in linear spaces with applications," Mathematical and Computer Modelling, vol. 52, no. 9-10, pp. 1497-1505, 2010.

[10] J. Rooin, "Some refinements of discrete Jensen's inequality and some of its applications," Nonlinear Functional Analysis and Applications, vol. 12, no. 1, pp. 107-118, 2007.

[11] L.-C. Wang, X.-F. Ma, and L.-H. Liu, "A note on some new refinements of Jensen's inequality for convex functions," Journal of Inequalities in Pure and Applied Mathematics, vol. 10, no. 2, article 48, 2009.

[12] X. L. Tang and J. J. Wen, "Some developments of refinned Jensen's inequality," Journal of Southwest University for Nationalities, vol. 29, pp. 20-26, 2003.

[13] S. Hussain and J. Pečarić, “An improvement of Jensen's inequality with some applications," Asian-European Journal of Mathematics, vol. 2, no. 1, pp. 85-94, 2009.

[14] G. Zabandan and A. Kılıçman, "A new version of Jensen's inequality and related results," Journal of Inequalities and Applications, vol. 2012, article 238, 2012.

[15] G. Zabandan and A. K1lıçman, "Several integral inequalities and an upper bound for the bidimensional Hermite-Hadamard inequality," Journal of Inequalities and Applications, vol. 2013, article 27, 2013. 


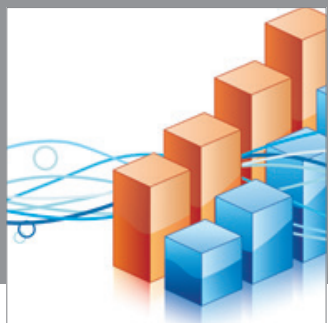

Advances in

Operations Research

mansans

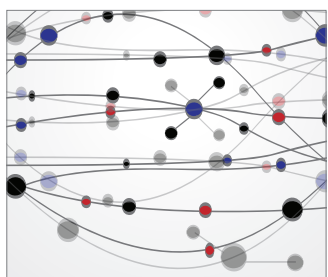

The Scientific World Journal
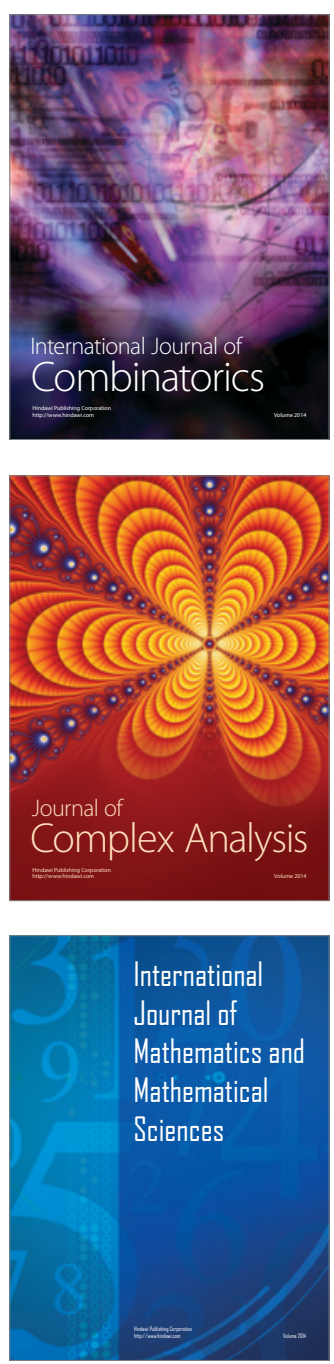
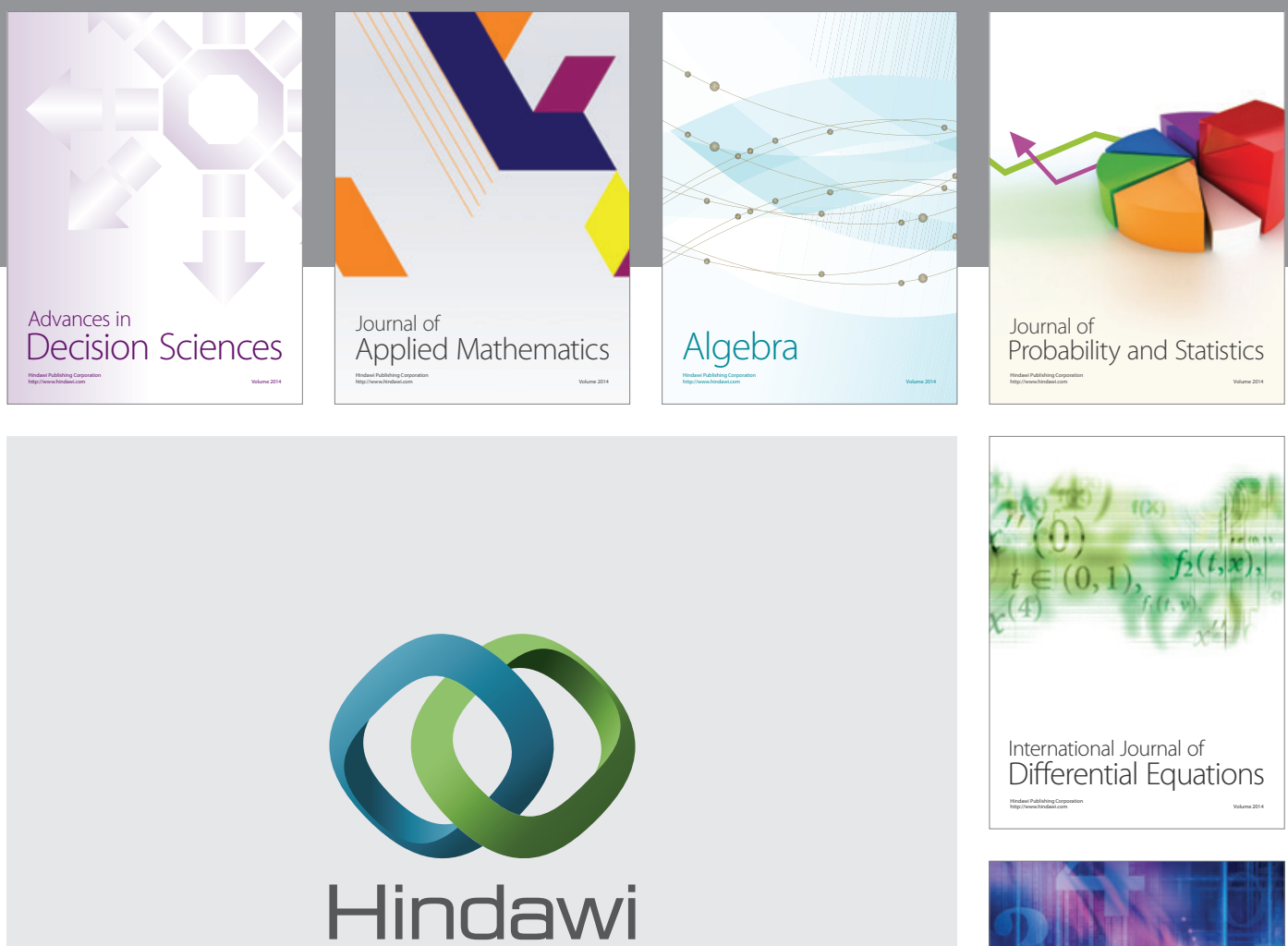

Submit your manuscripts at http://www.hindawi.com
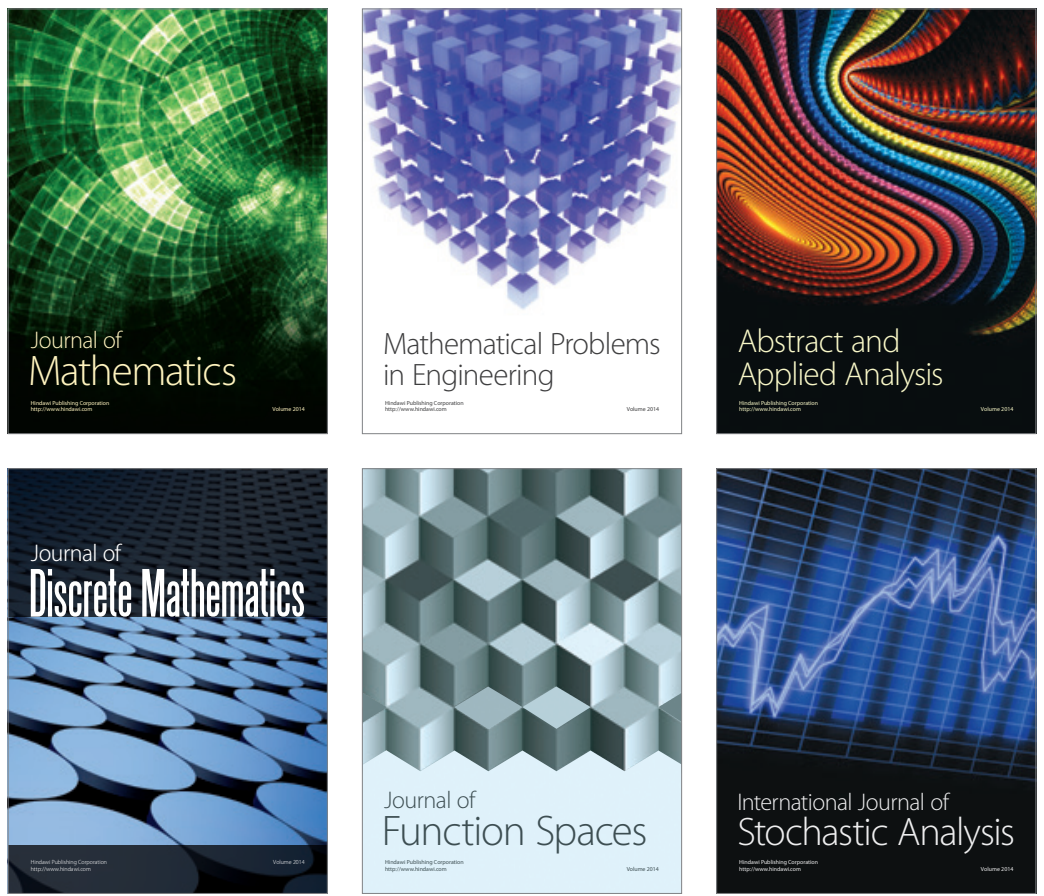

Journal of

Function Spaces

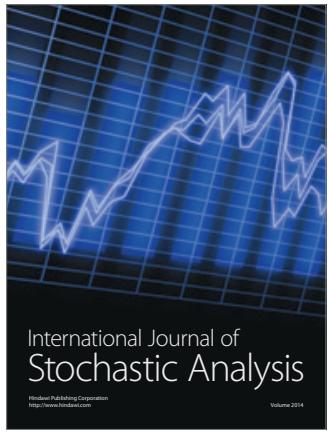

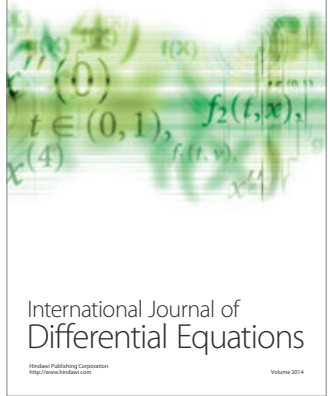
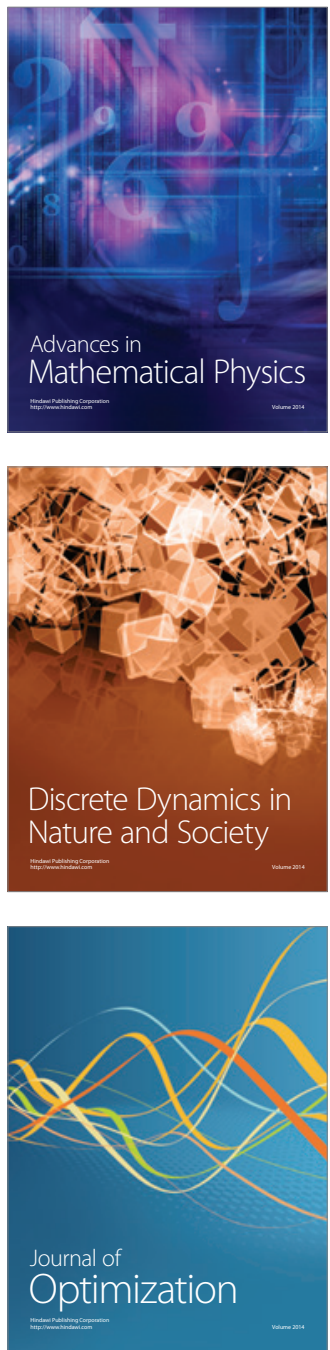